\title{
Hybrid Global Matrix/Local Interaction Simulation Approach for Wave Propagation Simulation in Composite Laminates
}

\author{
Matthew B. Obenchain, ${ }^{*}$ Kalyan S. Nadella, ${ }^{*}$ and Carlos E. S. Cesnik ${ }^{\dagger}$ \\ Department of Aerospace Engineering, University of Michigan, Ann Arbor, MI 48109, USA
}

\begin{abstract}
This paper presents a hybrid approach to model guided wave propagation in composite laminates. The global matrix approach is used to determine the displacement field surrounding a piezoelectric actuator. The displacement field is then enforced in a specified region of a numerical model that employs the local interaction simulation approach (LISA). This LISA Hybrid approach circumvents the problem of modeling non-rectangular actuators in the Cartesian discretization in LISA by using the global matrix method to characterize the actuator's influence on a cut-out region surrounding it. Results show the LISA Hybrid model outperforms previous LISA models that enforce in-plane displacements on the surface of the plate. The LISA Hybrid model produces wave propagation time histories that closely match the baseline global matrix method and successfully capture directional effects resulting from the anisotropic nature of composite plates. Results for aluminum, cross-ply, unidirectional, and quasi-isotropic plates show dependence on the in-plane discretization size, but that dependence is less pronounced for the cross-ply case.
\end{abstract}

\section{Introduction}

$\mathrm{U}$ SE of composite materials has proliferated in the aerospace community in recent years because of their significant advantages over conventional metals such as high specific strength, high specific stiffness, and long fatigue life. Appropriate structural health monitoring (SHM) strategies are required to ensure the functionality and reliability of composite structures. In the past, numerous studies ${ }^{1}$ have shown the use of guided waves $(\mathrm{GW})$ to be an efficient method for damage detection in metallic structures, which motivates similar research in the field of composite materials. Because of the anisotropic nature of composite materials, the development of SHM methods is significantly more complicated and challenging than in the case of isotropic materials. A comprehensive understanding of the GW propagation behavior is required to develop reliable structural health monitoring systems.

Previous researchers have modeled GW propagation in composites using a wide range of analytical, semianalytical, and numerical approaches. Purely analytical models for wave propagation in composites have been limited. For example, Ditri and Rose ${ }^{2}$ used a normal mode expansion technique to model excitation of anisotropic layers, but it was based on a 2-D elasticity model. Datta et al. ${ }^{3}$ highlighted the use of approximate plate theories due to the complexity of the equations obtained when a full 3-D theory of elasticity is used with layered plates. The most successful theoretical strategies for modeling wave propagation in multilayered plates have been the matrix approaches: the transfer matrix method and the global matrix method. ${ }^{4}$

Researchers have used the global matrix method as the basis for a semi-analytical formulation to study the GW excitation and propagation produced by finite-dimensional piezoelectric-based transducers. ${ }^{5-8}$ In the majority of these studies, the goal has been to use a theoretical approach to determine dispersion behavior or out-of-plane displacement values for comparison with experiments using laser vibrometry or other measurement techniques.

Although theoretical models are capable of characterizing the GW propagation in an infinite medium, they often become intractable when modeling complex composite structures because of the interface conditions and

${ }^{*} \mathrm{PhD}$ Candidate and AIAA Member

${ }^{\dagger}$ Professor and Fellow, AIAA; Corresponding Author; Email: cesnik@umich.edu; Phone: 1-734-764-3397; Fax: 1-734-7630578 
the need to account for the shape and reflections in the formulation. Researchers have turned to numerical simulations to facilitate the modeling of waves, which can better deal with complex structural systems, interfaces, and damage. ${ }^{9}$ Lee and Staszewski ${ }^{10}$ provided a good review of the many different numerical techniques used to model GW, including the finite difference (FD) approach, the finite element method, the boundary element method, and spectral element methods. While numerical techniques for modeling wave propagation are varied and have been successful, many have proved to be high in computational cost. ${ }^{1}$

The local interaction simulation approach (LISA), originally developed by Delsanto et al., ${ }^{11-13}$ has emerged as an efficient numerical option for modeling GW. This method is based on a set of iterative equations for unit cells that discretize the structure, and it uses a sharp interface model to handle material discontinuities and layer interfaces. Recent implementations ${ }^{14,15}$ of this method have shown good results, but some difficulties have surfaced. Notably, current LISA implementations have been limited to rectangular or cubic Cartesian grids, which create a challenge when modeling circular actuators. It would be advantageous to have a method that incorporates the behavior of the actuator without relying on the geometry of the grid in LISA.

The aim of this paper is to implement a hybrid approach to modeling wave propagation in composite laminates. The global matrix-based semi-analytical method is used to determine the complete displacement time history for a set of points located near a piezoelectric actuator both at the surface and through the thickness dimension of the composite plate. Subsequently, these known displacements are specified in LISA to represent the actuation pulse, and the remainder of the wave propagation model is obtained numerically. This provides a seamless way to integrate non-rectangular actuators into the Cartesian grid of the LISA simulation, allowing for an accurate representation of the wave field and avoiding errors associated with the actuator discretization.

\section{Global Matrix Solution}

\section{II.A. Theoretical Development}

Raghavan and Cesnik ${ }^{6}$ outlined a suitable framework to determine the displacements resulting from a piezoelectric actuator attached to the surface of a composite laminate. The starting point for the analysis is the displacement form of the equilibrium equation for a transversely isotropic bulk medium,

$$
\nabla \mathbf{S} \nabla^{T} \mathbf{u}=\rho \ddot{\mathbf{u}}
$$

where $\mathbf{S}$ is the stiffness matrix, $\mathbf{u}$ is the displacement vector, and $\rho$ is the density.

Assumption of a plane wave solution for Eq. 1 results in an eigenvalue problem that can be solved to yield

$$
\mathbf{u}=\left(C_{1}^{u} \mathbf{e}_{1} e^{i \zeta_{1} x_{3}}+C_{2}^{u} \mathbf{e}_{2} e^{i \zeta_{2} x_{3}}+C_{3}^{u} \mathbf{e}_{3} e^{i \zeta_{3} x_{3}}+C_{1}^{d} \mathbf{e}_{4} e^{-i \zeta_{1} x_{3}}+C_{2}^{d} \mathbf{e}_{5} e^{-i \zeta_{2} x_{3}}+C_{3}^{d} \mathbf{e}_{6} e^{-i \zeta_{3} x_{3}}\right) e^{-i\left(\xi_{1} x_{1}+\xi_{2} x_{2}-\omega t\right)}
$$

In this equation, $C_{i}^{u}$ and $C_{i}^{d}$ are constants associated with upward and downward traveling waves, respectively. Through-thickness wavenumbers are represented as $\zeta_{i}$, while in-plane wavenumbers are represented as $\xi_{i}$. The eigenvectors for the displacement field correspond to the $\mathbf{e}_{j}$ variables.

Based on the assumption that each composite ply is transversely isotropic, the global matrix approach introduced by Lih and $\mathrm{Mal}^{5}$ is used to account for the multilayer structure of the plate. A 2-D Fourier transform is used to move from the spatial domain to the wavenumber domain, and another Fourier transform is used to move from the time domain to the frequency domain. Coordinate transformations are used to convert local in-plane wavenumbers to global in-plane wavenumbers in polar coordinates. Subsequently, the global matrix is formed by enforcing displacement and stress continuity at the layer interfaces of the laminate. It is convenient to represent the stress and displacement quantities for a single lamina $m$ in vector form as

$$
\left[\begin{array}{cc}
L^{m} & 0 \\
0 & L^{m}
\end{array}\right]\left[\begin{array}{ll}
Q_{11}^{m} & Q_{12}^{m} \\
Q_{21}^{m} & Q_{22}^{m}
\end{array}\right]\left[\begin{array}{cc}
E_{u}^{m} & 0 \\
0 & E_{d}^{m}
\end{array}\right]\left[\begin{array}{c}
C_{u}^{m} \\
C_{d}^{m}
\end{array}\right]=Q^{m} C^{m}
$$

where $L^{m}$ is the transformation matrix corresponding to the orientation of the $m$ th lamina. The $Q_{i j}^{m}$ terms represent submatrices containing the stress and displacement eigenvectors in the local coordinate system. The contents of these submatrices can be found in detail in Refs. 5 and 6 . The $E$ terms are the exponentials 
from Eq. 2. The displacements and the three stresses contained in this vector are assumed to be continuous at the lamina interface between the bottom of lamina $m$ and the top of lamina $m+1$,

$$
Q_{\text {Bottom }}^{m} C^{m}=Q_{\text {Top }}^{m+1} C^{m+1}
$$

and the interface between lamina $m$ and lamina $m+1$ can be represented as

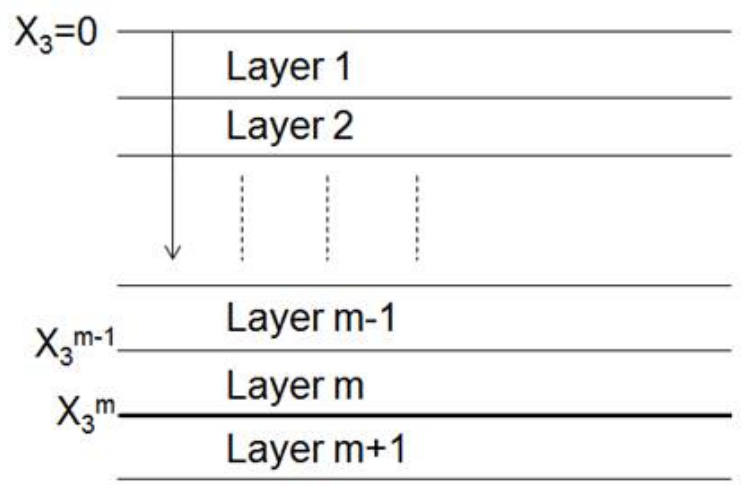

Figure 1. Schematic of laminated plate structure.

$$
\left[\begin{array}{ll}
Q_{\text {Bottom }}^{m} & -Q_{\text {Top }}^{m+1}
\end{array}\right]\left[\begin{array}{c}
C^{m} \\
C^{m+1}
\end{array}\right]=\left[\begin{array}{l}
0 \\
0
\end{array}\right]
$$

Figure 1 shows the layer numbering scheme used in this formulation. Applying this relationship to each interface results in the formation of the banded global matrix, which completely represents the laminated plate structure. The global matrix will have size $6 N \times 6 N$, where $N$ is the number of layers in the plate. When actuators are present on the top or bottom surface of the plate, these can be added to the forcing vector on the right hand side of

$$
\left[\begin{array}{ccccccccc}
\hat{Q}_{+}^{1} & 0 & \ldots & & & & & & \\
Q_{-}^{1} & Q_{+}^{2} & 0 & \ldots & & & & & \\
& & \ddots & & & & & & \\
& \cdots & 0 & Q_{-}^{m-1} & Q_{+}^{m} & 0 & \ldots & & \\
& & \cdots & 0 & Q_{-}^{m} & Q_{+}^{m+1} & 0 & \cdots & \\
& & & & & & \ddots & & \\
& & & & & \cdots & 0 & Q_{-}^{N-1} & Q_{+}^{N} \\
& & & & & & \cdots & 0 & \hat{Q}_{-}^{N}
\end{array}\right]\left[\begin{array}{c}
C^{1} \\
C^{2} \\
\\
\\
\\
\\
\\
\\
\\
\end{array}\right]=\left[\begin{array}{c}
F \\
0 \\
\\
\vdots \\
\\
\\
\\
\end{array}\right]
$$

Admissible in-plane wavenumbers are those that result in the determinant of the global matrix having a zero value. These wavenumbers can be used to calculate the admissible phase velocities and form dispersion curves for the plate. On the other hand, a complete solution of Eq. 6 is needed to determine the displacement solution for the plate. Determination of the $C_{i}^{u}$ and $C_{i}^{d}$ constants requires the forcing vector for the actuator to be represented in terms of a 2-D spatial Fourier transform. For the circular actuator, the transformed forcing vector has previously ${ }^{8}$ been shown to be:

$$
\begin{aligned}
& F_{1}=-i \tau_{0} R J_{1}(K R) \cos \Gamma \\
& F_{2}=-i \tau_{0} R J_{1}(K R) \sin \Gamma
\end{aligned}
$$

where $\tau_{0}$ is an amplitude constant for the stress transferred to the plate, $R$ is the radius of the actuator, $K$ is the global radial wavenumber, and $J_{1}$ represents the first-order Bessel function of the first kind. In this work, only a circular actuator will be considered. However, any actuator can be modeled using this approach as long as the tractions it produces can be represented in the wavenumber domain. Previous works have demonstrated the use of rectangular, ring, and CLoVER actuators. ${ }^{6}$ Cramer's rule provides a convenient way to determine the constant values once the forcing vector is in wavenumber space. 
Having determined the constants for each lamina, it is now possible to determine the displacements at any point on the plate. As mentioned earlier, most previous implementations of this method have only allowed for determination of the out-of-plane displacement component at the top surface of the plate. The hybrid model described in this work requires that all three displacement components be specified at certain locations in the model. For this reason, previous implementations have been extended to calculate all three displacement components. Additionally, capability has been added to determine displacement components at all lamina interface locations, not just the plate surface. The displacement vector at a given point on the plate is represented in the wavenumber and frequency domain as

$$
\mathbf{U}\left(K_{1}, K_{2}, X_{3}, \omega\right)=\left[\begin{array}{ll}
Q_{11}^{m} & Q_{12}^{m}
\end{array}\right]\left[\begin{array}{cc}
E_{u} & 0 \\
0 & E_{d}
\end{array}\right]\left[\begin{array}{c}
C_{u}^{m} \\
C_{d}^{m}
\end{array}\right]
$$

where $K_{1}$ and $K_{2}$ are the in-plane components of the global wavenumber, $X_{3}$ is the through-thickness location of the point of interest in global coordinates, $\omega$ is the frequency, and $Q_{11}^{m}$ and $Q_{12}^{m}$ are the displacement eigenvectors for the lamina containing the point of interest. The $C$ constants used in the calculation also correspond the lamina containing the point of interest.

The matrix of exponentials above must be calculated based on the through-thickness location of the point of interest. This matrix is in terms of the applicable lamina's local coordinate system and is given by:

$$
\left[\begin{array}{cc}
E_{u} & 0 \\
0 & E_{d}
\end{array}\right]=\left[\begin{array}{cccccc}
e^{i \zeta_{1} x_{3}} & 0 & 0 & 0 & 0 & 0 \\
0 & e^{i \zeta_{2} x_{3}} & 0 & 0 & 0 & 0 \\
0 & 0 & e^{i \zeta_{3} x_{3}} & 0 & 0 & 0 \\
0 & 0 & 0 & e^{-i \zeta_{1} x_{3}} & 0 & 0 \\
0 & 0 & 0 & 0 & e^{-i \zeta_{2} x_{3}} & 0 \\
0 & 0 & 0 & 0 & 0 & e^{-i \zeta_{3} x_{3}}
\end{array}\right]
$$

Once the matrices in Eq. 8 are calculated, they can be multiplied to determine the value of the displacement vector at the given wavenumber, through-thickness location, and frequency. It can be shown that this results in an expression for the out-of-plane displacement of the form:

$$
W\left(K_{1}, K_{2}, X_{3}, \omega\right)=R J_{1}(R K) \frac{\tau_{0}}{4 \pi^{2}} \frac{\Psi\left(K, \Gamma, X_{3}, \omega\right)}{\Delta(K, \Gamma, \omega)}
$$

where the $\Delta$ term represents the determinant of the global matrix. The $\Psi$ term is a function of the azimuthal wavenumber and the constants associated with the layer of interest. The other displacement components are formed similarly. In order to determine the values of the displacement component in the spatial and time domains, a series of inverse transformations is necessary. First, a 2-D inverse Fourier transform is used to move from the wavenumber domain to the spatial domain. The inversion formula is given by:

$$
W\left(r, \theta, X_{3}, \omega\right)=\int_{0}^{\infty} \int_{0}^{2 \pi} R J_{1}(K R) \frac{\tau_{0}}{4 \pi^{2}} \frac{\Psi\left(K, \Gamma, X_{3}, \omega\right)}{\Delta(K, \Gamma, \omega)} e^{-i K r \cos (\theta-\Gamma)} K d \Gamma d K
$$

In Eq. 11, the integrand is singular at wavenumber values that make the determinant of the global matrix zero. These are exactly the values of the propagating wavenumbers for the plate. Previous works ${ }^{6,8,16}$ have shown the employment of the residue formula to perform the complex integration needed to overcome this difficulty and complete the inversion. The result of the 2-D inversion is the displacement harmonic for the given spatial location and frequency. Finally, an inverse Fourier transform is used to move from the frequency domain to the time domain. The nature of the actuation must be considered during this step. In this work, a Hann-modulated toneburst signal with a given center frequency is used to excite the plate. An example with a $75 \mathrm{KHz}$ center frequency is shown in Fig. 2. To determine the frequency spectrum of the displacement at a given point, the Fourier transform of the actuation signal is multiplied by the displacement harmonic at each frequency. The inverse transform then produces the displacement time history at the point of interest.

\section{II.B. Solution Implementation}

In practice, the majority of this solution method is implemented in FORTRAN 90, which assembles the global matrix, determines the propagating wavenumbers for the laminate, and determines the values of the 

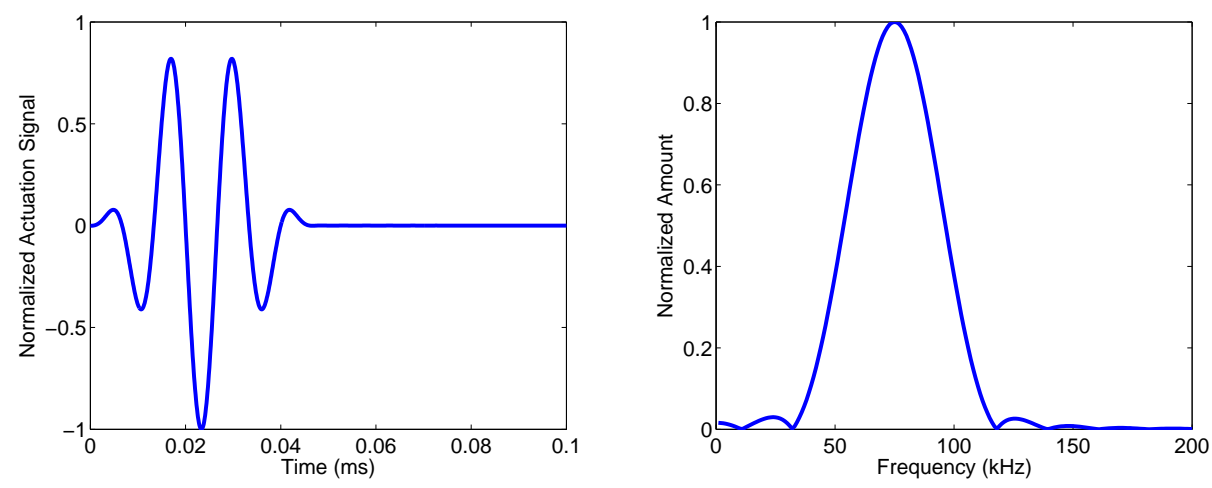

Figure 2. Actuation signal time history and frequency content.

integrand in Eq. 11. A MATLAB script is used to carry out the numerical integrations needed to complete the Fourier inversions and calculate the physical displacement values. Figure 3 shows the out-of-plane displacement time histories of the fundamental symmetric $\left(S_{0}\right)$ and antisymmetric $\left(A_{0}\right)$ modes at various ply interfaces resulting from a circular actuator mounted on a 12-layer cross-ply graphite-epoxy laminate $\left([0 / 90]_{3 s}\right)$ with a $1.5 \mathrm{~mm}$ thickness and material properties shown in Table 1 . These displacements are observed $50 \mathrm{~mm}$ from the center of the actuator. As one would expect, corresponding interfaces in the upper and lower halves of the plate have opposite out-of-plane displacements in the $S_{0}$ mode. All interfaces have similar out-of-plane displacements in the $A_{0}$ mode, which corresponds to the flexural nature of this mode.
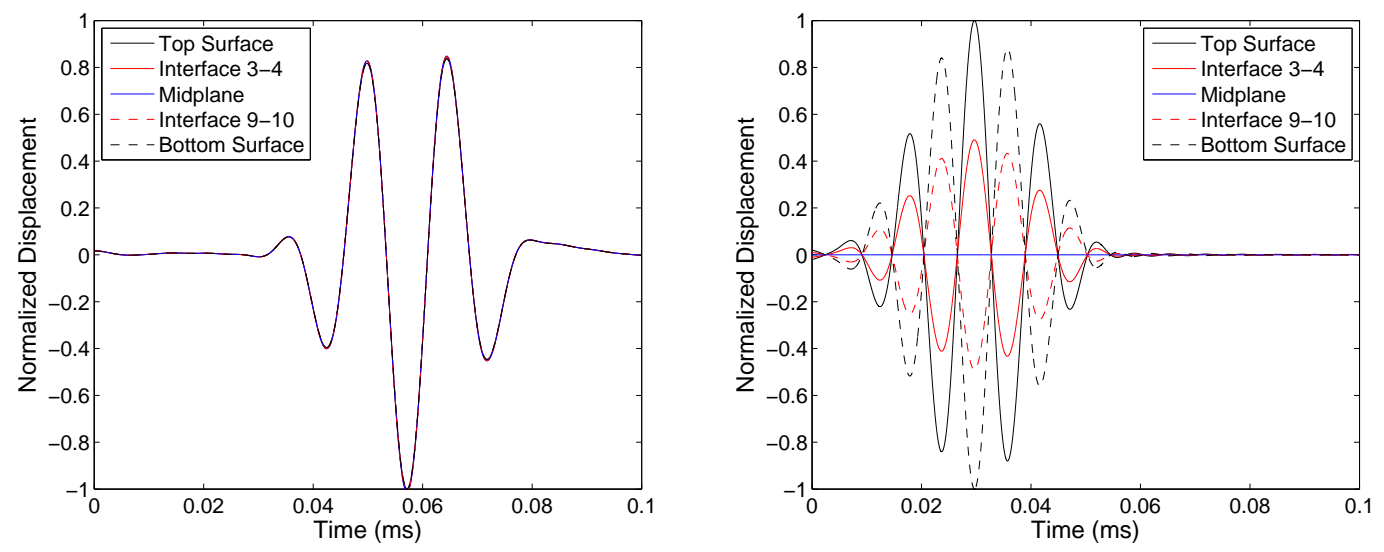

Figure 3. Global matrix displacement time histories for the fundamental antisymmetric (left) and symmetric (right) propagation modes in a cross-ply laminate. Displacements are observed $50 \mathrm{~mm}$ from the actuator center along the 0-degree direction.

Table 1. Graphite-epoxy lamina material properties

\begin{tabular}{|l||c|c|c|c|c|c|}
\hline Property & $E_{1}$ & $E_{2}$ & $G_{12}$ & $G_{23}$ & $\nu_{12}$ & $\rho$ \\
\hline Value & $147 \mathrm{GPa}$ & $9.8 \mathrm{GPa}$ & $2.35 \mathrm{GPa}$ & $3.3 \mathrm{GPa}$ & 0.405 & $1558 \mathrm{~kg} / \mathrm{m}^{3}$ \\
\hline
\end{tabular}

Table 2. Aluminum material properties

\begin{tabular}{|l||c|c|c|}
\hline Property & $E$ & $\nu$ & $\rho$ \\
\hline Value & $70 \mathrm{GPa}$ & 0.3 & $2700 \mathrm{~kg} / \mathrm{m}^{3}$ \\
\hline
\end{tabular}

In this paper, results from the global matrix method are used as the baseline case for pristine plates. Previous works have shown good correlation between experimental and global matrix results. To reiterate 
this point, Fig. 4 compares results from the global matrix model used in this paper and experimental results. Out-of-plane results are shown for points located 20,50, and $80 \mathrm{~mm}$ from the center of the circular actuator mounted on the top surface of an aluminum plate. The aluminum plate had a 3.18 mm thickness and the material properties shown in Table 2. In each case, the global matrix results closely match those from the experiment. The global matrix thus serves as a suitable baseline against which to judge the effectiveness of other wave propagation models.
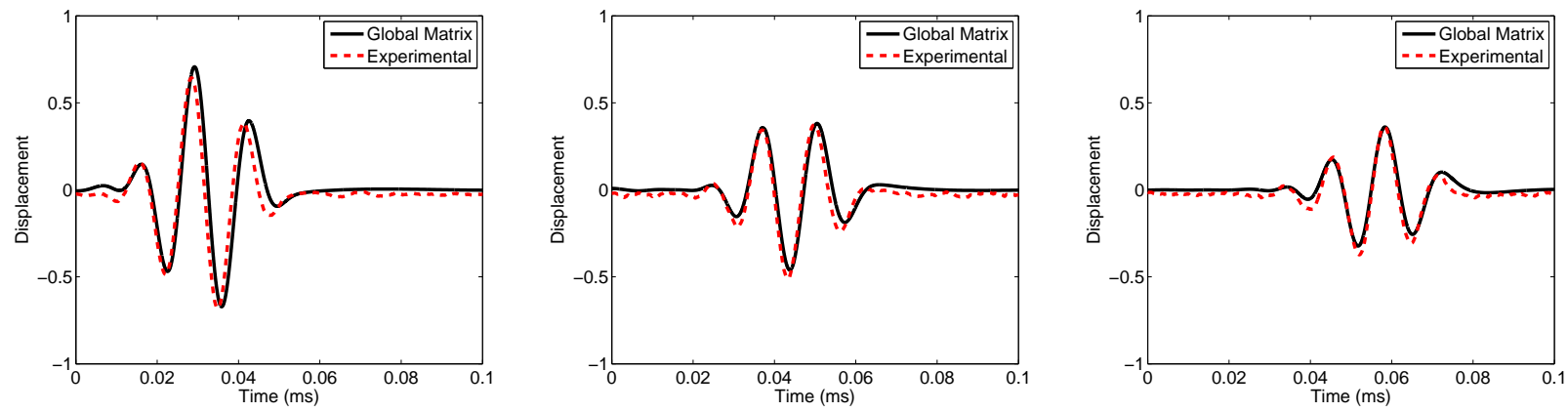

Figure 4. Comparison of global matrix and experimental out-of-plane displacement results for an aluminum plate with a circular PZT actuator on the top surface.

\section{Local Interaction Simulation Approach}

\section{III.A. Theoretical Development}

While the global matrix method can accurately capture wave propagation for a pristine plate with no lateral boundaries, numerical approaches are needed to capture features present in realistic structures. Modeling wave propagation using the finite element method has been a popular technique, but it is often inefficient due to the computational cost involved in resolving short wavelengths with small time increments. Delsanto et al. ${ }^{11-13}$ introduced an attractive alternative, LISA. This method is based on iterative equations (IE) for unit cells that are used to represent/discretize the model. The actual IEs are derived from the elastodynamic equilibrium equations ${ }^{15}$ in Eq. 1. Finite difference approximations form the backbone of the derivation, which can be seen in detail in several previous papers. ${ }^{9,13,17}$ Figure 5 summarizes the typical formulation process for LISA.

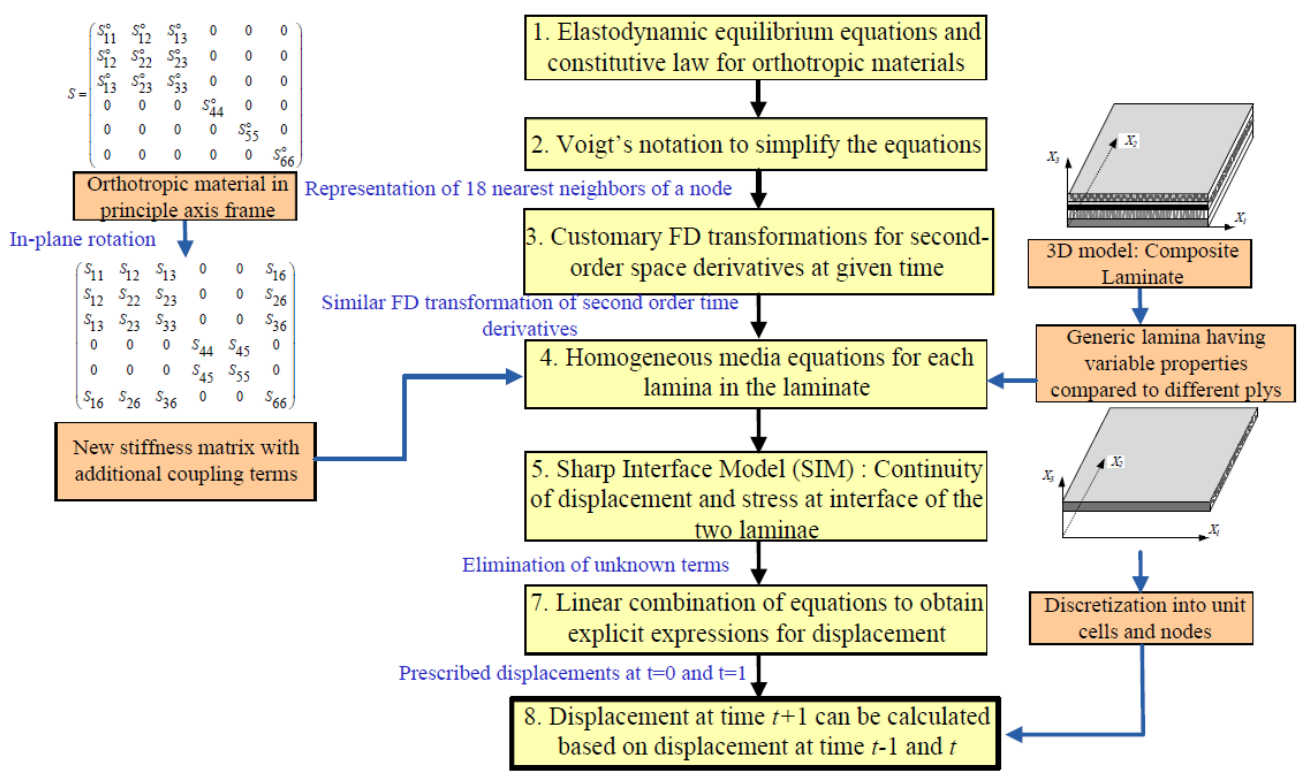

Figure 5. Overview of LISA theoretical formulation. ${ }^{15}$ 


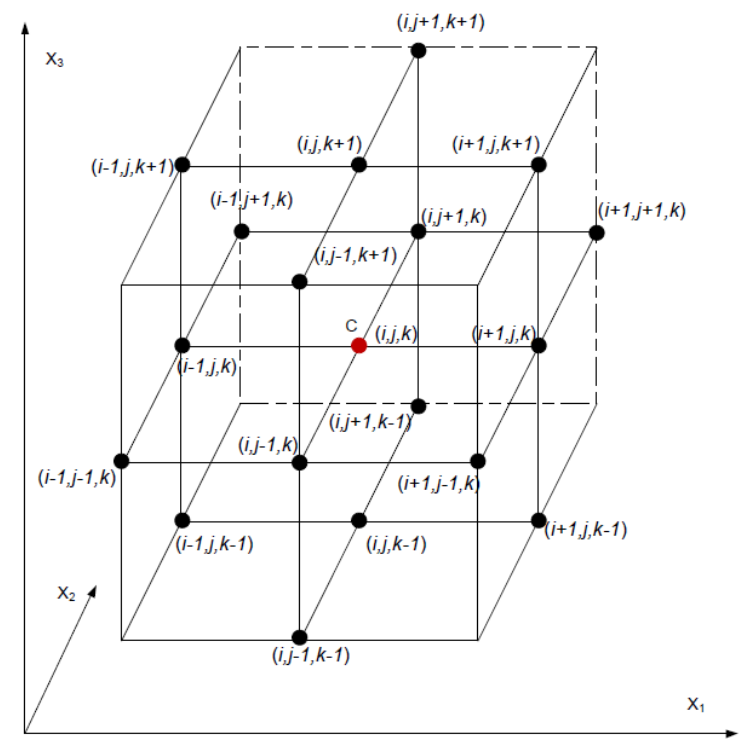

Figure 6. Representation of the 18 neighbors of generic point in LISA and the Cartesian system used in the analysis. ${ }^{15}$

The coefficients in the LISA iterative equations depend only on the local physical properties. LISA utilizes a sharp interface model where the actual conditions enforced at the cell interfaces are continuity of displacements and stresses. Therefore, changes in stiffness, density, or attenuation can be accounted for since the IE coefficients in adjacent cells with different properties will vary. The resulting formulation determines the response of any point at the current time step based on the response of that point's 18 nearest neighbors (shown in Fig. 6) in previous time steps.

Lee and Staszewski ${ }^{10,14,18,19}$ showed the utility of LISA for isotropic plates and extended their work to model simple damage in those plates using a two-dimensional model. Sundararaman and Adams used a three-dimensional LISA formulation to study the effect of simple damage in isotropic and unidirectional orthotropic plates ${ }^{9}$ and subsequently examined the effects of grid size. ${ }^{20}$ Recently, Nadella and Cesnik ${ }^{15,21}$ extended the approach to simulate wave propagation in arbitrary composite laminates and expanded the 3 -D formulation to account for plies in non-principal directions. The resulting iterative equations are used in this work, and they are included for reference in the appendix. Their work has previously demonstrated LISA's usefulness in modeling a variety of composite configurations including unidirectional, cross-ply, and quasi-isotropic laminates. In those studies, actuation was modeled by specifying in-plane displacements at surface nodes located within the profile of the actuator. A schematic of this actuation method for a circular PZT actuator is shown in Fig. 7.
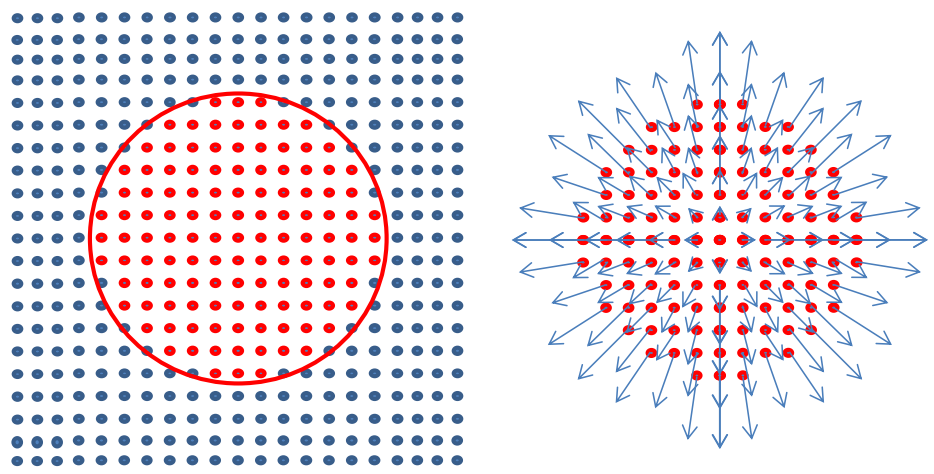

Figure 7. Original LISA actuation scheme using specified in-plane surface displacements. 


\section{III.B. Preliminary Numerical Results}

A series of models were evaluated using this actuation approach for an aluminum plate, a cross-ply plate $\left([0 / 90]_{3 s}\right)$, a unidirectional plate, and a quasi-isotropic plate $\left([0 / 30 / 60 /-60 /-30 / 90]_{s}\right)$. Each plate had a thickness of $1.5 \mathrm{~mm}$, and the composite plates contained 12 layers of the graphite-epoxy lamina from Table 1. Material properties for the aluminum plate are shown in Table 2. Results from these models are compared with results produced using the global matrix approach, since that approach has previously been shown to produce results that agree well with experiments. In the LISA models, a cell size of $0.25 \times 0.25 \times 0.125$ $\mathrm{mm}$ was used along with a time step of $10 \mathrm{~ns}$. These parameters meet the Courant-Friedric-Lewy (CFL) criterion, which is a necessary condition for stability for FD-based numerical schemes. ${ }^{9}$

$$
\mathrm{CFL}=c_{\max } \Delta t \sqrt{\frac{1}{\Delta x_{1}^{2}}+\frac{1}{\Delta x_{2}^{2}}+\frac{1}{\Delta x_{3}^{2}}} \leq 1
$$

As defined in Eq. 12, the CFL number must remain less than 1, and it is dependent on the time step used in the model as well as the spatial discretization. The $c_{\max }$ term represents the maximum propagating wave velocity. Traction-free boundary conditions were implemented using a layer of air cells surrounding the plate.

Results for the aluminum plate model are shown in Fig. 8. The time histories predicted at three different radial distances from the center of the actuator are shown for the global matrix model and the LISA model, henceforth referred to as the LISA original model. At each of the three locations it can be seen that the LISA original results show some discrepancies from the baseline global matrix results. Most notably, the duration of the pulse in the LISA model seems to be shorter than that in the global matrix results, as indicated by the Hilbert transform envelope included in the figures.
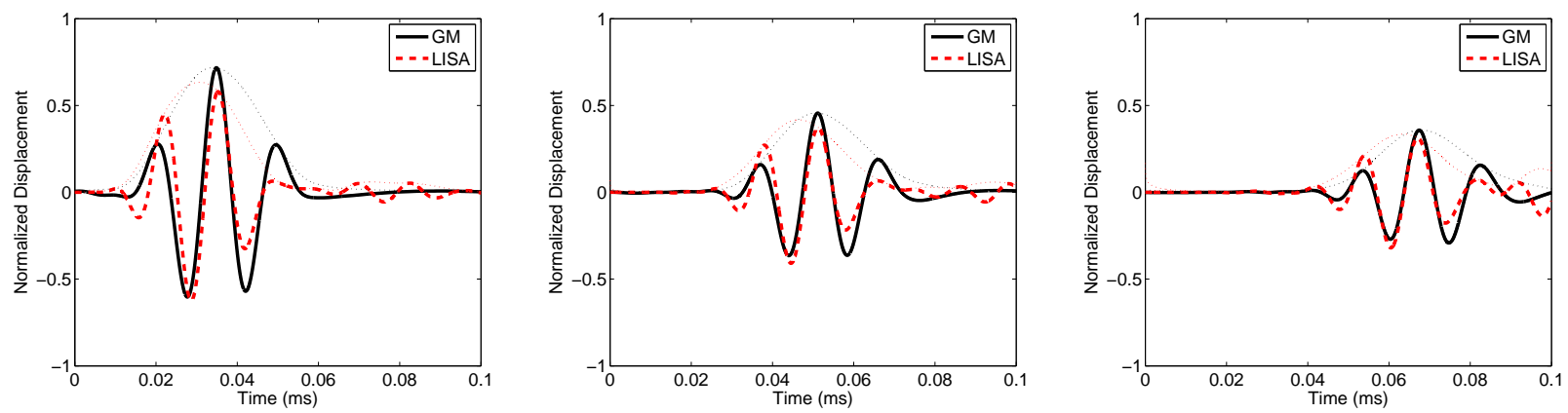

Figure 8. Comparison of global matrix and LISA original time histories for an aluminum plate. Locations 20, 50, and $80 \mathrm{~mm}$ from the actuator center are shown from left to right.

Results from the three composite models are shown in Figs. 9-11. Each of the time histories is for a point $40 \mathrm{~mm}$ from the actuator center. Results at 0,45 , and 90 degrees are presented since the results are not axisymmetric like in the aluminum case. The ability of the LISA original model to capture the wave propagation time histories is even worse for these models where the group velocities and attenuation vary with azimuthal direction.
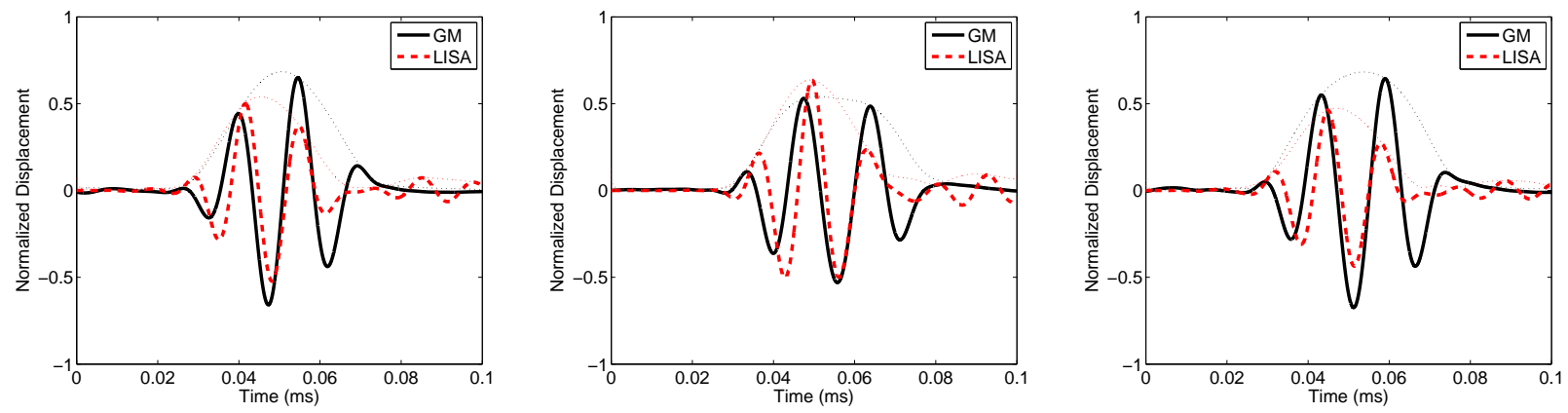

Figure 9. Comparison of global matrix and LISA original time histories for a cross-ply plate 40mm from the actuator center. The 0,45 , and 90-degree directions are shown from left to right. 

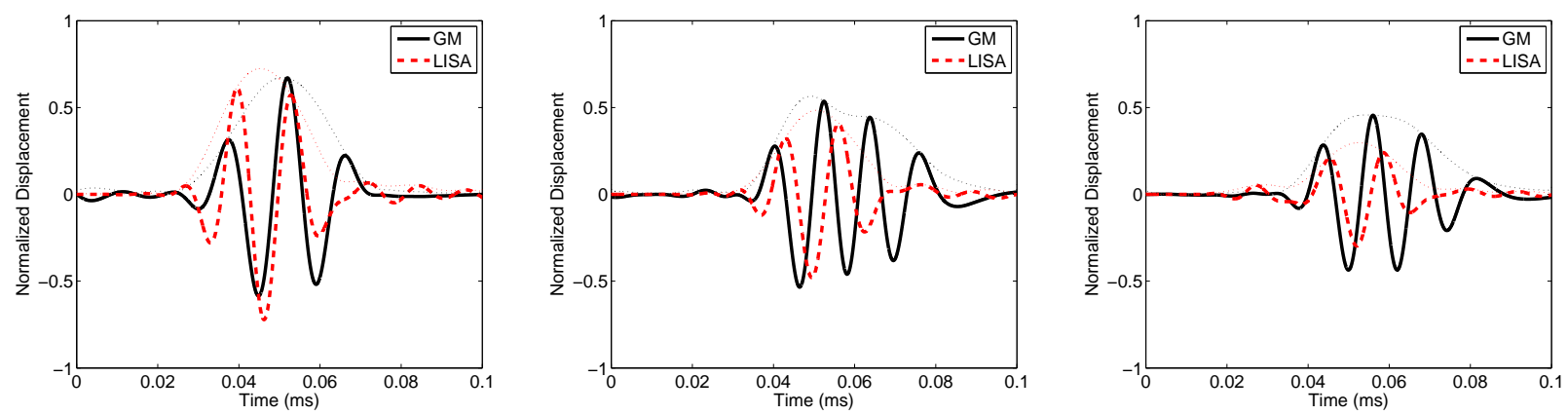

Figure 10. Comparison of global matrix and LISA original time histories for a unidirectional plate 40mm from the actuator center. The 0,45 , and 90-degree directions are shown from left to right.
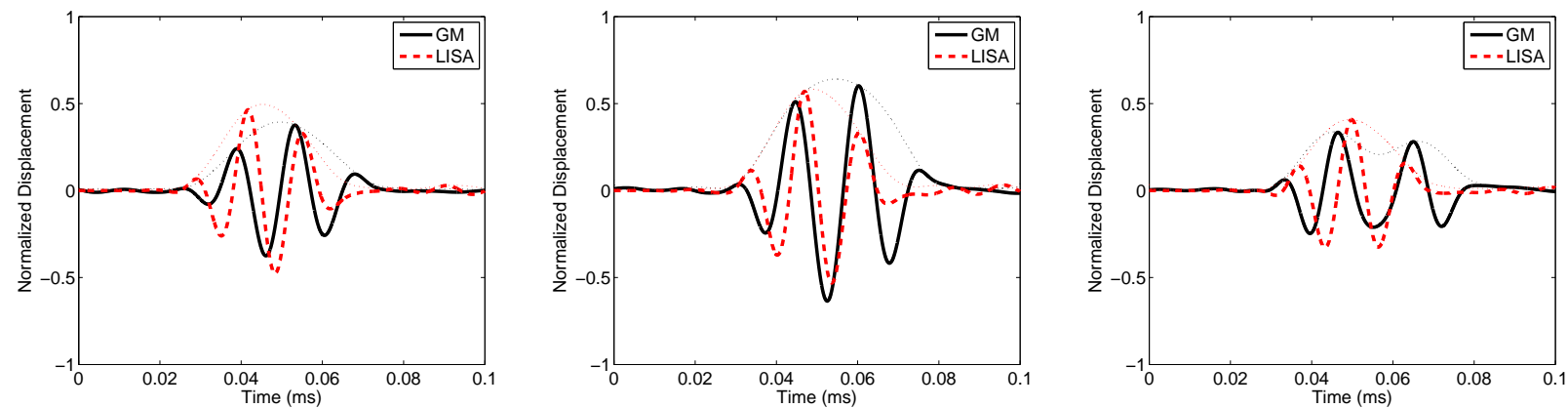

Figure 11. Comparison of global matrix and LISA original time histories for a quasi-isotropic plate 40mm from the actuator center. The 0,45 , and 90-degree directions are shown from left to right.

Looking at the previous results and the actuation diagram in Fig. 7, it is evident that the current method of specifying in-plane displacements on the plate surface to represent the actuator is not completely successful. The cuboid nature of the LISA grid forces the user to decide how best to approximate the nonlinear profile of a non-rectangular actuator. The boundary of the actuator must be approximated by locating the closest nodes. This problem can be mitigated with a finer discretization, but only if the user accepts the corresponding computational cost. Even with a finer discretization, this method still relies on the imposition of specified displacements on the surface of the plate, which was shown to not accurately model the piezoelectric effect. ${ }^{22}$

\section{Hybrid Model}

The global matrix method is well suited to capture the effects of finite-dimensional actuators, but it cannot capture damage or lateral plate boundaries. On the other hand, LISA can successfully model a finite-dimensional plate, but using non-rectangular actuators can be challenging. As an alternative, a LISA Hybrid model is introduced that leverages the strengths of both methods. It uses the global matrix approach to represent the effects of the actuator while using LISA for the majority of the plate structure. First, the overall LISA model is defined according to the structure of the plate, including any material differences between layers for a laminate. As in previous LISA models, air cells are included at the boundary to allow for traction-free boundary conditions. Next, a cut-out region around the actuator is defined as in Fig. 12, and the nodes within the LISA model located on the boundary of this region are identified and tabulated.

Third, the time histories of the three displacement components resulting from the piezoelectric actuation are calculated at each of the cut-out boundary points using the global matrix method. Because the entire time history of the displacements is calculated and stored, this step need only to be run once for the plate configuration used in the simulation. Finally, the square cut-out region surrounding the actuator is segregated from the remainder of the model, as shown in Fig. 13, and the LISA simulation is started. At each time step in the LISA simulation, the displacement values for the boundary nodes of this region are specified according to the global matrix time history. The LISA model is run as usual to completely capture the wave 


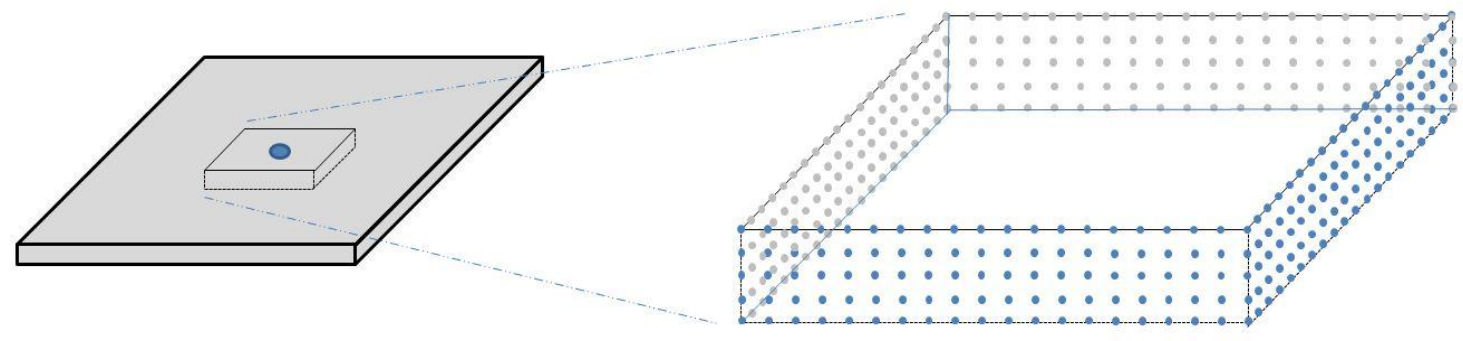

Figure 12. Schematic of displacement input field for LISA Hybrid wave propagation model.

propagation behavior for the remainder of the plate.

While the LISA Hybrid model still specifies displacements, the actuation modeling method is distinct from previous LISA implementations. Because the LISA Hybrid model uses the global matrix method to capture the behavior of the actuator, it assumes the actuator imposes tractions, not displacements, on the plate surface. These tractions are included in the solution process used to determine the displacement constants in Eq. 6, which are then used to determine the displacement time histories at all points along cut-out boundary.

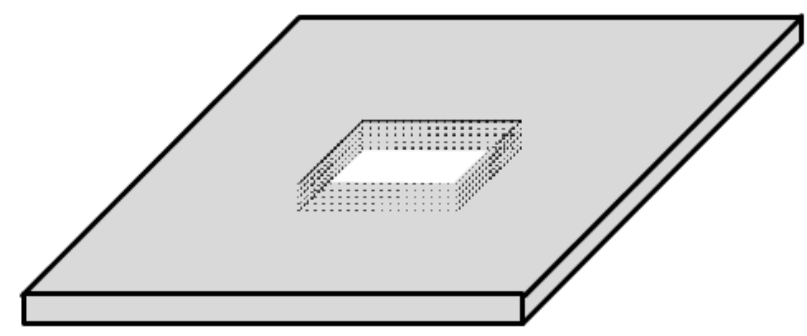

Figure 13. Schematic of LISA Hybrid plate model showing boundary of "cut-out" region.

\section{IV.A. LISA Hybrid Results}

The LISA Hybrid model was run for the four plates analyzed in the previous section. In these models, a cell size of $0.25 \times 0.25 \times 0.125 \mathrm{~mm}$ was used to discretize the model. The cut-out region was defined as a 20 by $20 \mathrm{~mm}$ square. Since the displacements on the boundary of the cut-out are specified as the global matrix displacements, the hybrid model will exactly match the global matrix results for all points located on this boundary. If the LISA Hybrid model is successful in capturing the propagating wave, then the model results will also match the global matrix results at locations further from the actuator. The results for the aluminum model are shown in Fig. 14. The results included at 20, 50, and $80 \mathrm{~mm}$ indicate that this is indeed the case.

For the three composite models, the LISA Hybrid results are compared at the 0, 45, and 90-degree directions to examine the models' effectiveness in various azimuthal directions. Each point of interest is 40 $\mathrm{mm}$ from the actuator center. Figures 15 and 16 show excellent agreement between the LISA Hybrid results and the baseline global matrix results for the cross-ply and unidirectional plates.

In Fig. 17 it can be seen that the LISA Hybrid model slightly overestimates the amplitude of the propagating wave in the 0 and 90-degree directions, but the results for 45 degrees show excellent agreement The LISA Hybrid model represents the propagation velocity and general "character" of the wave very well in each of the three composite cases examined in this work.

To better see the effectiveness of the LISA Hybrid model in capturing the directional dependence of the wave propagation in a composite plate, Fig. 18 includes field plots of the out-of-plane displacement of 

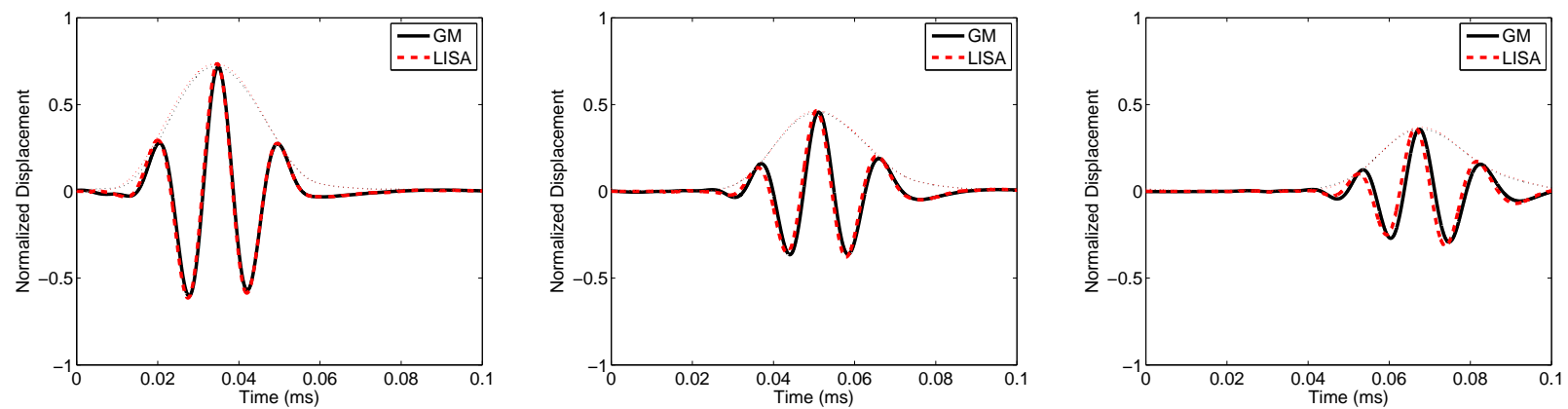

Figure 14. Comparison of global matrix and LISA Hybrid time histories for an aluminum plate. Locations 20, 50, and $80 \mathrm{~mm}$ from the actuator center are shown from left to right.
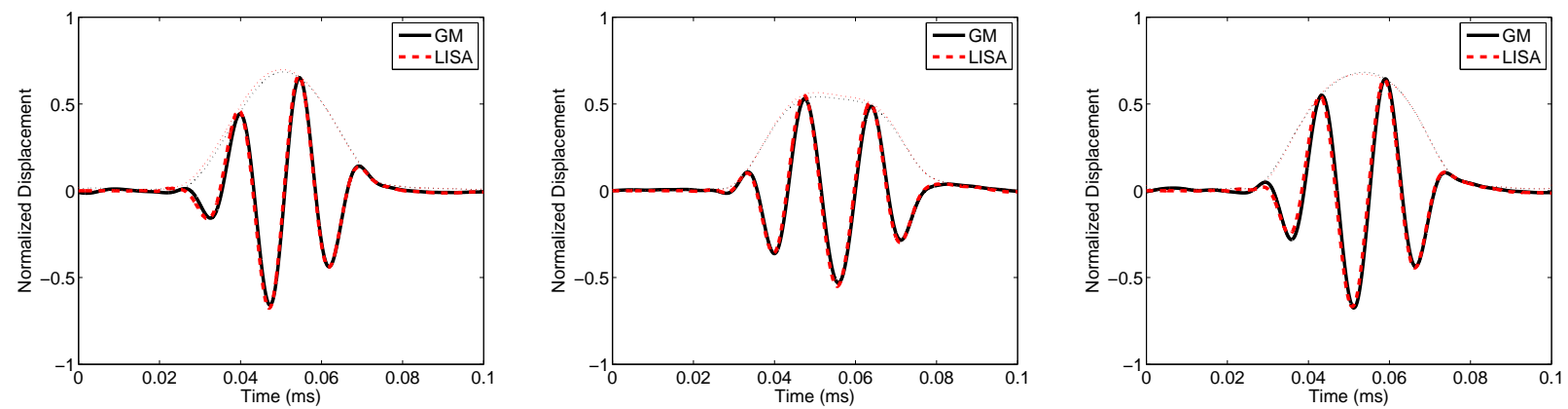

Figure 15. Comparison of global matrix and LISA Hybrid time histories for a cross-ply plate 40mm from the actuator center. The 0, 45, and 90-degree directions are shown from left to right.
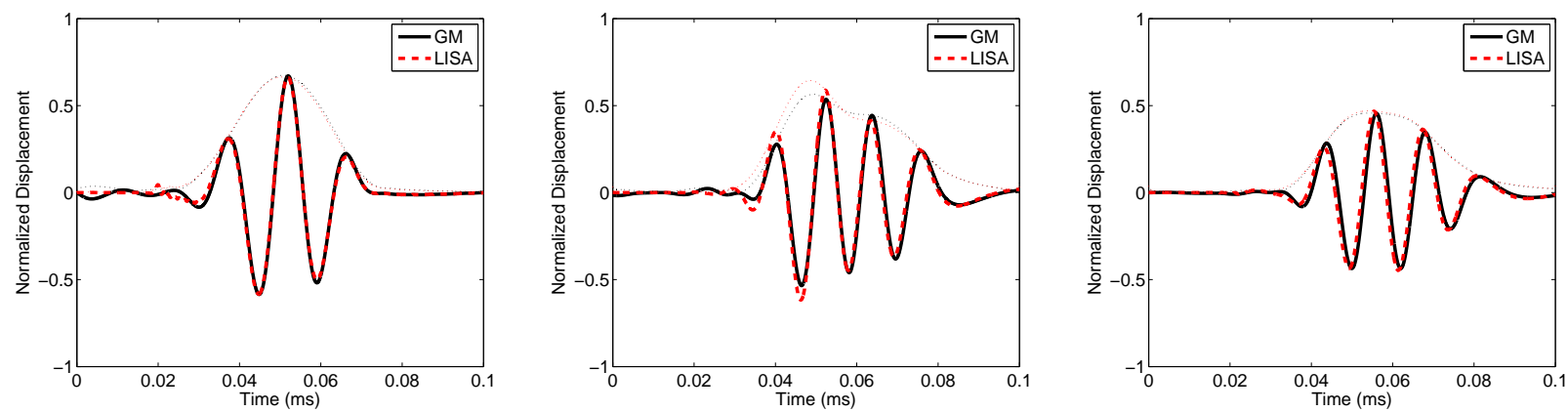

Figure 16. Comparison of global matrix and LISA Hybrid time histories for a unidirectional plate 40mm from the actuator center. The 0,45 , and 90-degree directions are shown from left to right.
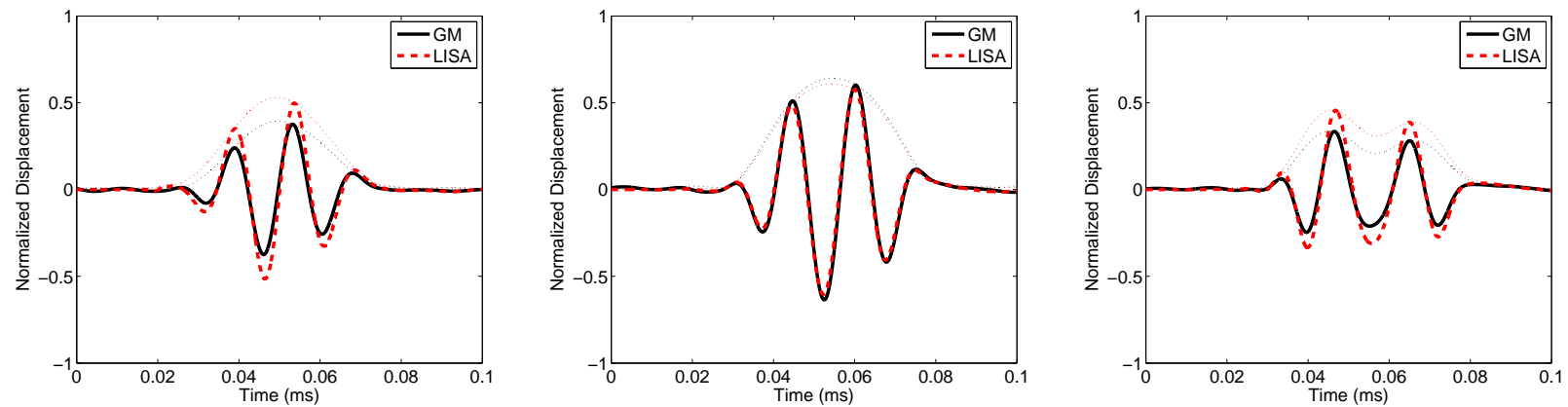

Figure 17. Comparison of global matrix and LISA Hybrid time histories for a quasi-isotropic plate 40mm from the actuator center. The 0,45 , and 90-degree directions are shown from left to right. 
the cross-ply plate. The global matrix and LISA Hybrid results are compared at 40,60 and $80 \mu$ s. The preponderance of energy propagating in the 0 and 90-degree directions is preserved well in the new modeling approach.

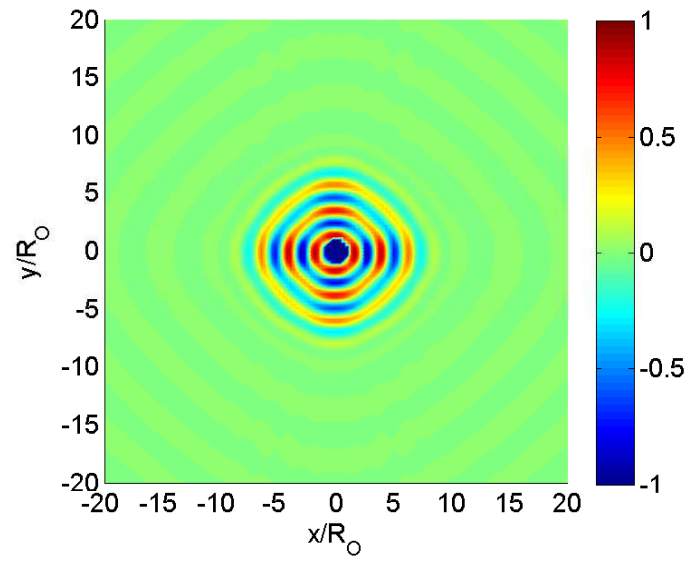

(a) GM $40 \mu \mathrm{s}$

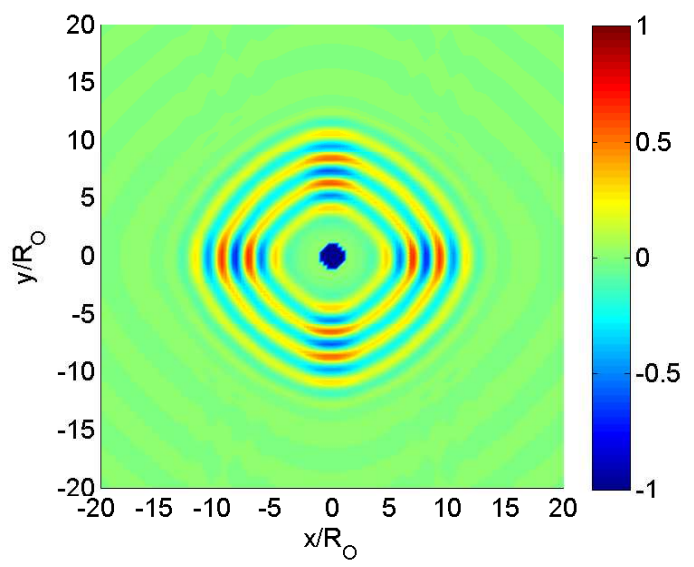

(c) GM $60 \mu \mathrm{s}$

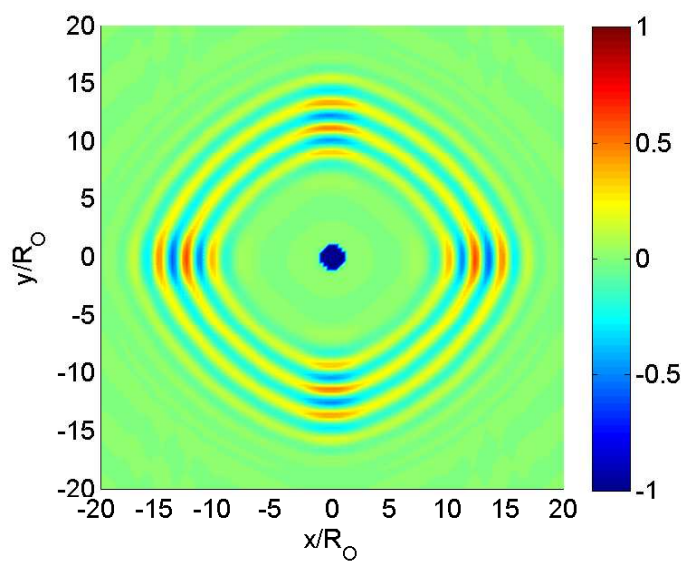

(e) GM $80 \mu \mathrm{s}$

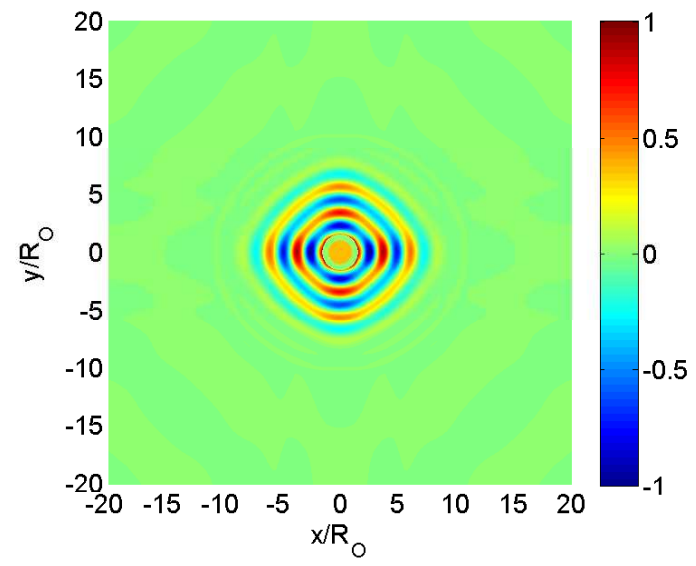

(b) LISA Hybrid $40 \mu \mathrm{s}$

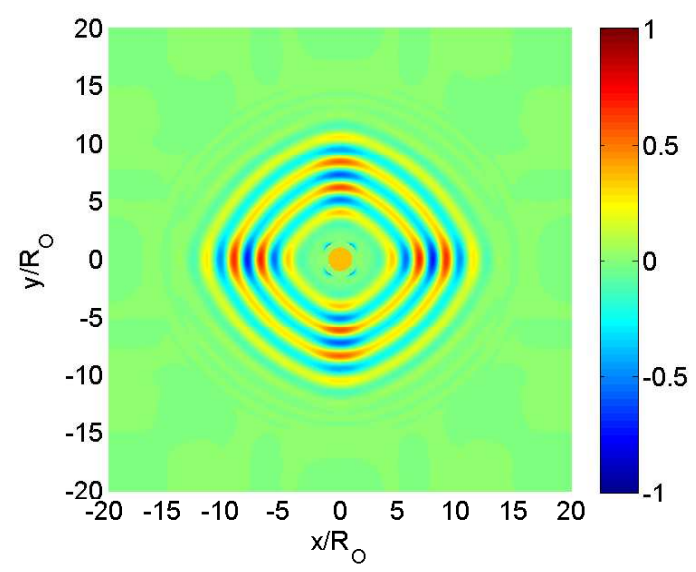

(d) LISA Hybrid $60 \mu \mathrm{s}$

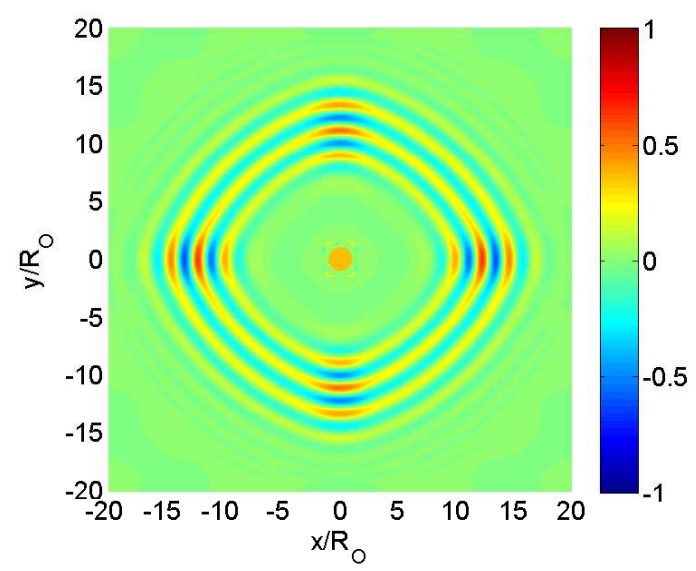

(f) LISA Hybrid $80 \mu \mathrm{s}$

Figure 18. Comparison of global matrix and LISA Hybrid results for out-of-plane displacement of a cross-ply plate at various times. 


\section{IV.B. Effect of In-plane Discretization}

Previous researchers ${ }^{20}$ have noted the dependence of LISA results on the in-plane discretization. This dependence is also seen in the present LISA Hybrid results, particularly in the group velocity of the propagating wave. The four plate models were run with in-plane cell sizes of 1.0, 0.5 , and $0.25 \mathrm{~mm}$. The through-thickness discretization was held constant at $0.125 \mathrm{~mm}$. The results indicate the group velocity predicted by the LISA Hybrid model increases as the in-plane cell size increases. This trend was very noticeable for the aluminum plate. As shown in Fig. 19, the results for each of the three discretization sizes agree very will with the baseline model at $20 \mathrm{~mm}$. However, at more distant points the effect of the group velocity differences is more apparent. The plot for the $80 \mathrm{~mm}$ location demonstrates the effect of overly coarse discretizations on the model results.
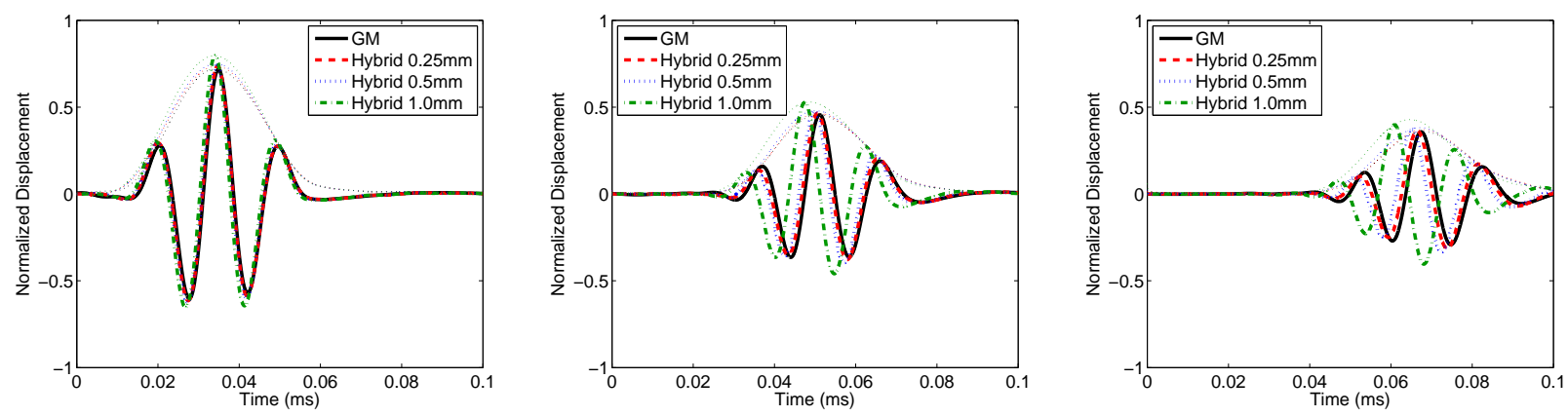

Figure 19. Comparison of various LISA Hybrid in-plane discretizations for an aluminum plate. Locations 20, 50, and $80 \mathrm{~mm}$ from the actuator center are shown.

This effect was also seen in the composite models, and not surprisingly the effect is direction dependent. The three discretization sizes were compared for the 0,45 , and 90-degree directions at points $70 \mathrm{~mm}$ from the actuator center. At this distance the group velocity differences have had time to manifest themselves. Compared to the aluminum model, the cross-ply model shown in Fig. 20 is much less sensitive to the cell size, but the results are affected more for the 90-degree direction than the 0-degree direction. For the unidirectional model, the directional dependence is even more pronounced, as seen in Fig. 21. Here all three cell sizes give good results at 0 degrees, but the results for coarse discretizations worsen as the azimuth increases from 0 to 90 degrees. Finally, Fig. 22 shows the results for the quasi-isotropic model.
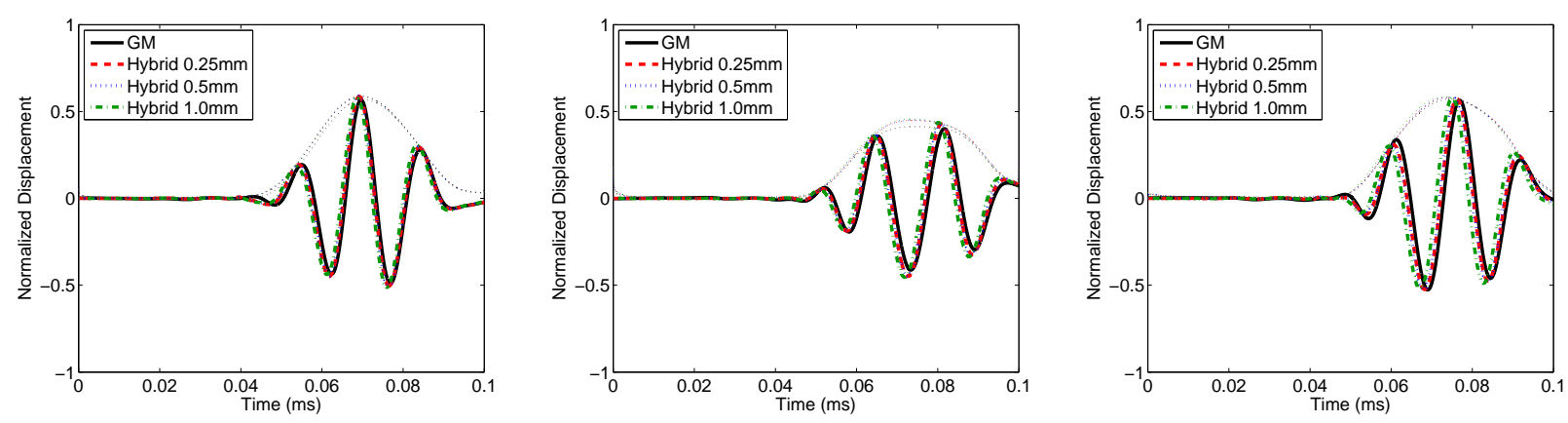

Figure 20. Comparison of various LISA Hybrid in-plane discretizations for the cross-ply laminate at a point 70 mm away from the actuator center. The 0,45 , and 90-degree directions are shown from left to right.

\section{Conclusion}

This work introduced a LISA Hybrid wave propagation model that takes advantage of the strengths of the global matrix method and LISA. The global matrix method was used to determine the effects of a circular actuator mounted on the top surface of a plate. The LISA formulation for composite plates with plies in non-principal directions was used to capture the wave propagation away from the actuator. The LISA Hybrid model was used to produce displacement results for an aluminum plate and three graphite-epoxy plates (cross-ply, unidirectional, and quasi-isotropic). These results were compared with baseline results 

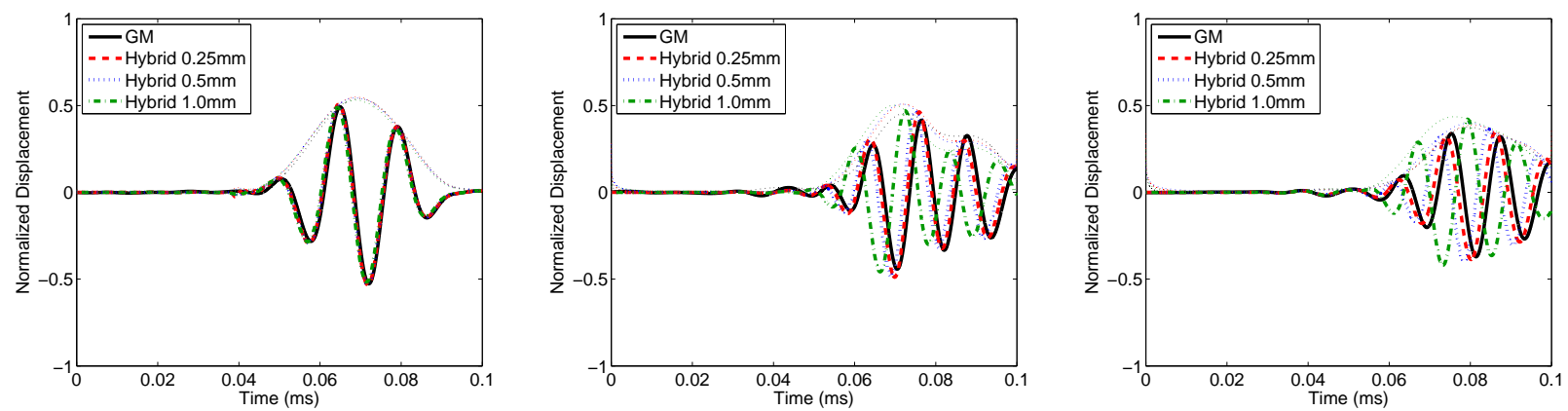

Figure 21. Comparison of various LISA Hybrid in-plane discretizations for the unidirectional laminate at a point 70 $\mathrm{mm}$ away from the actuator center. The 0,45 , and 90-degree directions are shown from left to right.
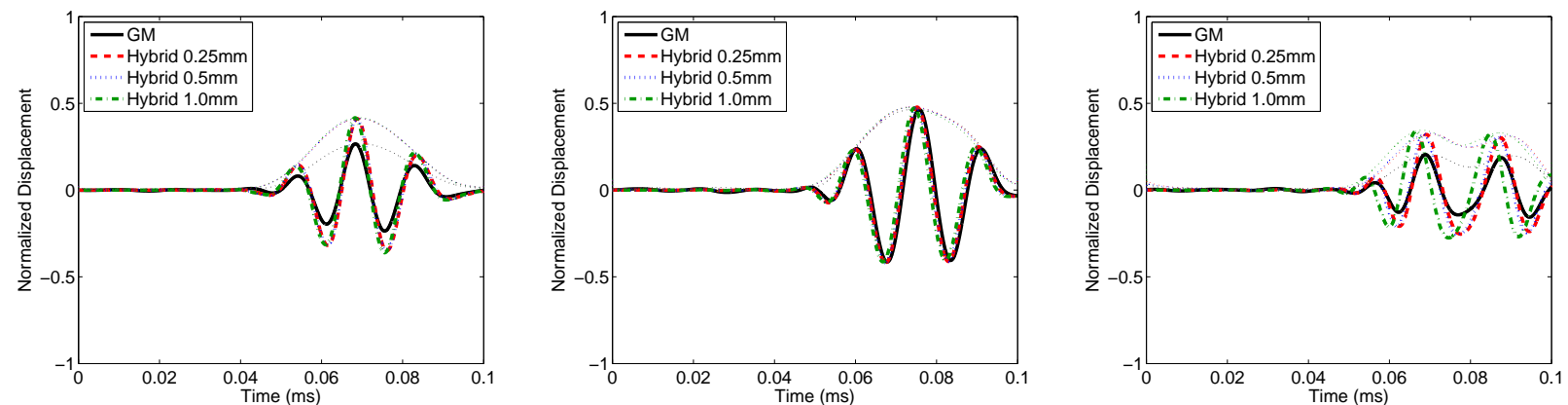

Figure 22. Comparison of various LISA Hybrid in-plane discretizations for the quasi-isotropic laminate at a point $\mathbf{7 0}$ $\mathrm{mm}$ away from the actuator center. The 0,45 , and 90-degree directions are shown from left to right.

from the global matrix method. In all cases, the LISA Hybrid results produced more accurate time histories than previous LISA models, and the results match those from the global matrix method extremely well. A brief study on the in-plane discretization size used in the LISA Hybrid model showed the importance of this parameter and its effect on the resulting displacement time histories. Consistent with previous work, coarse discretizations produced artificially fast propagating waves. Additionally, this work showed the influence of the azimuthal direction on the discretization effects. For all four models, a $\frac{1}{6} t \times \frac{1}{6} t \times \frac{1}{12} t$ cell size produced results agreeing well with the baseline model for plates of thickness $t$. With the LISA Hybrid model producing accurate results for the pristine plates considered in this work, the next step will be to use the model to consider various types of damage likely to be seen in realistic aerospace structures. 


\section{Appendix}

LISA iterative equations as derived by Nadella and Cesnik ${ }^{15}$ which allow for in-plane rotation of the orthotropic lamina.

$$
\begin{aligned}
& U^{i, j, k, t+1}=-U^{i, j, k, t-1}+2 U^{i, j, k}-\frac{2 \chi}{8} U^{i, j, k} \sum_{\alpha, \beta, \gamma= \pm 1}\left[\left(\eta_{x}^{2} \tilde{S}_{11}+\eta_{y}^{2} \tilde{S}_{66}+\eta_{z}^{2} \tilde{S}_{55}\right)\right] \\
& +\frac{\chi}{8} \sum_{\alpha, \beta, \gamma= \pm 1}\left[2 \eta_{x}^{2} \tilde{S}_{11} U^{i+\alpha, j, k}+2 \eta_{y}^{2} \tilde{S}_{66} U^{i, j+\beta, k}+2 \eta_{z}^{2} \tilde{S}_{55} U^{i, j, k+\gamma}\right] \\
& +\frac{\chi}{8} \sum_{\alpha, \beta, \gamma= \pm 1}\left[\alpha \beta \eta_{x} \eta_{y}\left(\tilde{S}_{12}+\tilde{S}_{66}\right)\left(V^{i+\alpha, j+\beta, k}-V^{i, j, k}\right)\right]+\frac{\chi}{8} \sum_{\alpha, \beta, \gamma= \pm 1}\left[\alpha \beta \eta_{x} \eta_{y}\left(\tilde{S}_{12}-\tilde{S}_{66}\right)\left(V^{i, j+\beta, k}-V^{i+\alpha, j, k}\right)\right] \\
& +\frac{\chi}{8} \sum_{\alpha, \beta, \gamma= \pm 1}\left[\alpha \gamma \eta_{x} \eta_{z}\left(\tilde{S}_{13}+\tilde{S}_{55}\right)\left(W^{i+\alpha, j, k+\gamma}-W^{i, j, k}\right)\right]+\frac{\chi}{8} \sum_{\alpha, \beta, \gamma= \pm 1}\left[\alpha \gamma \eta_{x} \eta_{z}\left(\tilde{S}_{13}-\tilde{S}_{55}\right)\left(W^{i, j, k+\gamma}-W^{i+\alpha, j, k}\right)\right] \\
& -\frac{2 \chi}{8} \sum_{\alpha, \beta, \gamma= \pm 1}\left[\alpha \beta \eta_{x} \eta_{y} \tilde{S}_{16}\left(U^{i, j, k}-U^{i+\alpha, j+\beta, k}\right)\right]-\frac{2 \chi}{8} V^{i, j, k} \sum_{\alpha, \beta, \gamma= \pm 1}\left[\eta_{x}^{2} \tilde{S}_{16}+\eta_{y}^{2} \tilde{S}_{26}\right] \\
& -\frac{\chi}{8} \sum_{\alpha, \beta, \gamma= \pm 1}\left[\beta \gamma \eta_{y} \eta_{z}\left(\tilde{S}_{36}+\tilde{S}_{45}\right) W^{i, j, k}\right]+\frac{2 \chi}{8} \sum_{\alpha, \beta, \gamma= \pm 1}\left[\eta_{x}^{2} \tilde{S}_{16} V^{i+\alpha, j, k}+\eta_{y}^{2} \tilde{S}_{26} V^{i, j+\beta, k}\right] \\
& +\frac{\chi}{8} \sum_{\alpha, \beta, \gamma= \pm 1}\left[\beta \gamma \eta_{y} \eta_{z} \tilde{S}_{36}\left(W^{i, j+\beta, k+\gamma}+W^{i, j, k+\gamma}-W^{i, j+\beta, k}\right)\right] \\
& +\frac{\chi}{8} \sum_{\alpha, \beta, \gamma= \pm 1}\left[\beta \gamma \eta_{y} \eta_{z} \tilde{S}_{45}\left(W^{i, j+\beta, k+\gamma}-W^{i, j, k+\gamma}+W^{i, j+\beta, k}\right)\right]+\frac{2 \chi}{8} \sum_{\alpha, \beta, \gamma= \pm 1} \eta_{z}^{2} \tilde{S}_{45}\left(V^{i, j, k+\gamma}-V^{i, j, k}\right) \\
& V^{i, j, k, t+1}=-V^{i, j, k, t-1}+2 V^{i, j, k} \\
& -\frac{2 \chi}{8} V^{i, j, k} \sum_{\alpha, \beta, \gamma= \pm 1}\left[\eta_{x}^{2} \tilde{S}_{66}+\eta_{y}^{2} \tilde{S}_{22}+\eta_{z}^{2} \tilde{S}_{44}\right]+\frac{\chi}{8} \sum_{\alpha, \beta, \gamma= \pm 1}\left[2 \eta_{x}^{2} \tilde{S}_{66} V^{i+\alpha, j, k}+2 \eta_{y}^{2} \tilde{S}_{22} V^{i, j+\beta, k}+2 \eta_{z}^{2} \tilde{S}_{44} V^{i, j, k+\gamma}\right] \\
& +\frac{\chi}{8} \sum_{\alpha, \beta, \gamma= \pm 1}\left[\alpha \beta \eta_{x} \eta_{y}\left(\tilde{S}_{12}+\tilde{S}_{66}\right)\left(U^{i+\alpha, j+\beta, k}-U^{i, j, k}\right)\right]+\frac{\chi}{8} \sum_{\alpha, \beta, \gamma= \pm 1}\left[\alpha \beta \eta_{x} \eta_{y}\left(\tilde{S}_{12}-\tilde{S}_{66}\right)\left(U^{i+\alpha, j, k}-U^{i, j+\beta, k}\right)\right] \\
& +\frac{\chi}{8} \sum_{\alpha, \beta, \gamma= \pm 1}\left[\beta \gamma \eta_{y} \eta_{z}\left(\tilde{S}_{23}+\tilde{S}_{44}\right)\left(W^{i, j+\beta, k+\gamma}-W^{i, j, k}\right)\right]+\frac{\chi}{8} \sum_{\alpha, \beta, \gamma= \pm 1}\left[\beta \gamma \eta_{y} \eta_{z}\left(\tilde{S}_{23}-\tilde{S}_{44}\right)\left(W^{i, j, k+\gamma}-W^{i, j+\beta, k}\right)\right] \\
& -\frac{2 \chi}{8} \sum_{\alpha, \beta, \gamma= \pm 1}\left[\alpha \beta \eta_{x} \eta_{y} \tilde{S}_{26}\left(V^{i, j, k}-V^{i+\alpha, j+\beta, k}\right)\right]-\frac{2 \chi}{8} U^{i, j, k} \sum_{\alpha, \beta, \gamma= \pm 1}\left[\eta_{x}^{2} \tilde{S}_{16}+\eta_{y}^{2} \tilde{S}_{26}\right] \\
& +\frac{2 \chi}{8} \sum_{\alpha, \beta, \gamma= \pm 1}\left[\eta_{x}^{2} \tilde{S}_{16} U^{i+\alpha, j, k}+\eta_{y}^{2} \tilde{S}_{26} U^{i, j+\beta, k}\right]-\frac{\chi}{8} \sum_{\alpha, \beta, \gamma= \pm 1}\left[\alpha \gamma \eta_{x} \eta_{z}\left(\tilde{S}_{36}+\tilde{S}_{45}\right) W^{i, j, k}\right] \\
& +\frac{\chi}{8} \sum_{\alpha, \beta, \gamma= \pm 1}\left[\alpha \gamma \eta_{x} \eta_{z} \tilde{S}_{36}\left(W^{i+\alpha, j, k+\gamma}+W^{i, j, k+\gamma}-W^{i+\alpha, j, k}\right)\right] \\
& +\frac{\chi}{8} \sum_{\alpha, \beta, \gamma= \pm 1}\left[\alpha \gamma \eta_{x} \eta_{z} \tilde{S}_{45}\left(W^{i+\alpha, j, k+\gamma}-W^{i, j, k+\gamma}+W^{i+\alpha, j, k}\right)\right]+\frac{2 \chi}{8} \sum_{\alpha, \beta, \gamma= \pm 1}\left[\eta_{z}^{2} \tilde{S}_{45}\left(U^{i, j, k+\gamma}-U^{i, j, k}\right)\right]
\end{aligned}
$$




$$
\begin{aligned}
& W^{i, j, k, t+1}=-W^{i, j, k, t-1}+2 W^{i, j, k}-\frac{2 \chi}{8} W^{i, j, k} \sum_{\alpha, \beta, \gamma= \pm 1}\left[\eta_{x}^{2} \tilde{S}_{55}+\eta_{y}^{2} \tilde{S}_{44}+\eta_{z}^{2} \tilde{S}_{33}\right] \\
& +\frac{\chi}{8} \sum_{\alpha, \beta, \gamma= \pm 1}\left[2 \eta_{x}^{2} \tilde{S}_{55} W^{i+\alpha, j, k}+2 \eta_{y}^{2} \tilde{S}_{44} W^{i, j+\beta, k}+2 \eta_{z}^{2} \tilde{S}_{33} W^{i, j, k+\gamma}\right] \\
& +\frac{\chi}{8} \sum_{\alpha, \beta, \gamma= \pm 1}\left[\beta \gamma \eta_{y} \eta_{z}\left(\tilde{S}_{23}+\tilde{S}_{44}\right)\left(V^{i, j+\beta, k+\gamma}-V^{i, j, k}\right)\right]+\frac{\chi}{8} \sum_{\alpha, \beta, \gamma= \pm 1}\left[\beta \gamma \eta_{y} \eta_{z}\left(\tilde{S}_{23}-\tilde{S}_{44}\right)\left(V^{i, j+\beta, k}-V^{i, j, k+\gamma}\right)\right] \\
& +\frac{\chi}{8} \sum_{\alpha, \beta, \gamma= \pm 1}\left[\alpha \gamma \eta_{x} \eta_{z}\left(\tilde{S}_{13}+\tilde{S}_{55}\right)\left(U^{i+\alpha, j, k+\gamma}-U^{i, j, k}\right)\right]+\frac{\chi}{8} \sum_{\alpha, \beta, \gamma= \pm 1}\left[\alpha \gamma \eta_{x} \eta_{z}\left(\tilde{S}_{13}-\tilde{S}_{55}\right)\left(U^{i+\alpha, j, k}-U^{i, j, k+\gamma}\right)\right] \\
& -\frac{\chi}{8} \sum_{\alpha, \beta, \gamma= \pm 1}\left[\beta \gamma \eta_{y} \eta_{z}\left(\tilde{S}_{36}+\tilde{S}_{45}\right)\left(U^{i, j, k}-U^{i, j+\beta, k+\gamma}\right)\right]-\frac{\chi}{8} \sum_{\alpha, \beta, \gamma= \pm 1}\left[\alpha \gamma \eta_{x} \eta_{z}\left(\tilde{S}_{36}+\tilde{S}_{45}\right)\left(V^{i, j, k}-V^{i+\alpha, j, k+\gamma}\right)\right] \\
& -\frac{\chi}{8} \sum_{\alpha, \beta, \gamma= \pm 1}\left[\beta \gamma \eta_{y} \eta_{z}\left(\tilde{S}_{36}-\tilde{S}_{45}\right)\left(U^{i, j, k+\gamma}-U^{i, j+\beta, k}\right)\right]-\frac{\chi}{8} \sum_{\alpha, \beta, \gamma= \pm 1}\left[\alpha \gamma \eta_{x} \eta_{z}\left(\tilde{S}_{36}-\tilde{S}_{45}\right)\left(V^{i, j, k+\gamma}-V^{i+\alpha, j, k}\right)\right] \\
& +\frac{2 \chi}{8} \sum_{\alpha, \beta, \gamma= \pm 1}\left[\alpha \beta \eta_{x} \eta_{y} \tilde{S}_{45}\left(W^{i+\alpha, j+\beta, k}-W^{i, j, k}\right)\right] \\
& \eta_{x}=1 / \Delta x_{1} \\
& \eta_{y}=1 / \Delta x_{2} \\
& \eta_{z}=1 / \Delta x_{3} \\
& \chi=\Delta t^{2} / \rho
\end{aligned}
$$

In these equations, the current time step $t$ is assumed when it is not specifically mentioned. Stiffness terms with a tilde, such as $\tilde{S}_{11}$, indicate that the value of that term is defined by the value for one of the eight surrounding cells, depending on the current value of $\alpha, \beta, \gamma$.

\section{Acknowledgments}

This work was sponsored by the National Rotorcraft Technology Center (NTRC) Vertical Lift/Rotorcraft Center of Excellence (VLRCOE) at the University of Michigan, with John Berry as the technical monitor. Opinions, interpretations, conclusions, and recommendations are those of the authors and are not necessarily endorsed by the United States Government.

\section{References}

\footnotetext{
${ }^{1}$ Raghavan, A. and Cesnik, C. E. S., "Review of Guided Wave Structural Health Monitoring," The Shock and Vibration Digest, Vol. 39, No. 2, 2007, pp. 91-144.

${ }^{2}$ Ditri, J. J. and Rose, J. L., "Excitation of Guided Waves in Generally Anisotropic Layers Using Finite Sources," Journal of Applied Mechanics, Vol. 61, 1994, pp. 330-338.

${ }^{3}$ Datta, S. K., Shah, A. H., Bratton, R. L., and Chakraborty, T., "Wave Propagation in Laminated Composite Plates," Journal of the Acoustical Society of America, Vol. 83, No. 6, 1988, pp. 2020-2026.

${ }^{4}$ Lowe, M. J. S., "Matrix Techniques for Modeling Ultrasonic Waves in Multilayered Media," IEEE Transactions on Ultrasonics, Ferroelectrics, and Frequency Control, Vol. 42, No. 4, 1995, pp. 525-542.

${ }^{5}$ Lih, S. S. and Mal, A. K., "On the Accuracy of Approximate Plate Theories for Wave Field Calculations in Composite Laminates," Wave Motion, Vol. 21, 1995, pp. 17-34.

${ }^{6}$ Raghavan, A. and Cesnik, C. E. S., "Modeling of Guided-wave Excitation by Finite-dimensional Piezoelectric Transducers in Composite Plates," Proceedings of the 48th AIAA/ASME/ASCE/AHS/ASC Structures, Structural Dynamics, and Materials Conference, Honolulu, Hawaii, 2007.

${ }^{7}$ Salas, K. I. and Cesnik, C. E. S., "Guided Wave Structural Health Monitoring using CLoVER Transducer in Composite Plates," Proceedings of the 50th AIAA/ASME/ASCE/AHS/ASC Structures, Structural Dynamics, and Materials Conference, Palm Springs, California, 2009.

${ }^{8}$ Nadella, K. S., Salas, K. I., and Cesnik, C. E. S., "Characterization of Guided-wave Propagation in Composite Plates," Proceedings of the SPIE 17th International Symposium on Smart Structures and Materials + Nondestructive Evaluation and Health Monitoring, San Diego, California, Vol. 7650, 2010.
} 
${ }^{9}$ Sundararaman, S. and Adams, D. E., "Modeling Guided Waves for Damage Identification in Isotropic and Orthotropic Plates Using a Local Interaction Simulation Approach," Journal of Vibration and Acoustics.

${ }^{10}$ Lee, B. C. and Staszewski, W. J., "Modeling of Lamb Waves for Damage Detection in Metallic Structures: Part I. Wave Propagation," Smart Materials and Structures, Vol. 12, No. 5, 2003, pp. 804-814.

${ }^{11}$ Delsanto, P. P., Whitcombe, T., Chaskelis, H. H., and Mignogna, R. B., "Connection Machine Simulation of Ultrasonic Wave Propagation in Materials I: the One-dimensional Case," Wave Motion, Vol. 16, 1992, pp. 65-80.

${ }^{12}$ Delsanto, P. P., Schechter, R. S., Chaskelis, H. H., Mignogna, R. B., and Kline, R., "Connection Machine Simulation of Ultrasonic Wave Propagation in Materials II: the Two-dimensional Case," Wave Motion, Vol. 20, 1994, pp. $295-314$.

${ }^{13}$ Delsanto, P. P., Schechter, R. S., and Mignogna, R. B., "Connection Machine Simulation of Ultrasonic Wave Propagation in Materials III: the Three-dimensional Case," Wave Motion, Vol. 26, 1997, pp. 329-339.

${ }^{14}$ Lee, B. C. and Staszewski, W. J., "Modeling of Lamb Waves for Damage Detection in Metallic Structures: Part II. Wave Interactions with Damage," Smart Materials and Structures, Vol. 12, No. 5, 2003, pp. 815-824.

${ }^{15}$ Nadella, K. S. and Cesnik, C. E. S., "Numerical Simulation of Wave Propagation in Composite Plates," Proceedings of the SPIE 19th International Symposium on Smart Structures and Materials + Nondestructive Evaluation and Health Monitoring, San Diego, California, Vol. 8348, 2012.

${ }^{16}$ Banerjee, S., Prosser, W., and Mal, A., "Calculation of the Response of a Composite Plate to Localized Dynamic Surface Loads Using a New Wave Number Integral Method," Journal of Applied Mechanics, Vol. 72, No. 1, 2005, pp. 18-24.

${ }^{17}$ Sinor, M., "Numerical Modeling and Visualization of Elastic Waves Propagation in Arbitrary Complex Media," 8th Workship on Multimedia in Physics Teaching and Learning of the European Physical Society, Graz, 2004.

${ }^{18}$ Lee, B. C. and Staszewski, W. J., "Lamb Wave Propagation Modeling for Damage Detection: I. Two-dimensional Analysis," Smart Materials and Structures, Vol. 16, No. 5, 2007, pp. 249-259.

${ }^{19}$ Lee, B. C. and Staszewski, W. J., "Lamb Wave Propagation Modeling for Damage Detection: II. Damage Monitoring Strategy," Smart Materials and Structures, Vol. 16, No. 5, 2003, pp. 260-274.

${ }^{20}$ Sundararaman, S. and Adams, D. E., "Accuracy and Convergence Using a Local Interaction Simulation Approach in One, Two, and Three Dimensions," Journal of Applied Mechanics, Vol. 76, No. 3, 2009, pp. 031008.

${ }^{21}$ Nadella, K. S. and Cesnik, C. E. S., "Simulation of Guided Wave Propagation in Isotropic and Composite Structures using LISA," Proceedings of the 53rd AIAA/ASME/ASCE/AHS/ASC Structures, Structural Dynamics, and Materials Conference, Honolulu, Hawaii, 2012.

${ }^{22}$ Nadella, K. S. and Cesnik, C. E. S., "Piezoelectric Coupled LISA for Guided Wave Generation and Propagation," Proceedings of the SPIE 20th International Symposium on Smart Structures and Materials + Nondestructive Evaluation and Health Monitoring, San Diego, California, Vol. 8695, 2013. 